\title{
Imaging of Magnetic Domains and Domain Walls in Spherical Fe-Si Powder Using Magnetic Force Microscopy
}

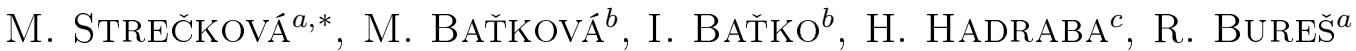 \\ ${ }^{a}$ Institute of Materials Research, Slovak Academy of Sciences, Watsonova 47, 04353 Košice, Slovakia \\ ${ }^{b}$ Institute of Experimental Physics, Slovak Academy of Sciences, Watsonova 47, 04001 Košice, Slovakia \\ ${ }^{c}$ CEITEC IPM, Institute of Physics of Materials, Academy of Sciences, Žižkova 22, Brno, Czech Republic
}

The commercial Fe-Si powder, produced by Högänes Corporation, represents promising soft magnetic material for technological applications. The powder consists of spherical particles with diameter up to $150 \mu \mathrm{m}$. Internal microstructure of the powder is formed by grains of diameter of about $30 \mu \mathrm{m}$. Each separate grain has a random orientation of the easy magnetization axis and is sufficiently large to split into several magnetic domains. A comparative study of the atomic force microscopy (AFM) topography and the corresponding magnetic force microscopy (MFM) images was employed in order to examine the correlation between the grain size, boundaries of grains and characterization of the magnetic domains, which gives us an important knowledge about possible behavior of particles under the influence of the external magnetic field and further utilization of the spherical Fe-Si particles in electrotechnical industry. The grain size and the crystallographic orientation of grains were analyzed by the electron backscattering diffraction (EBSD) technique.

DOI: 10.12693/APhysPolA.126.92

PACS: $75.50 . \mathrm{Bb}, 75.60 . \mathrm{Ch}, 75.70 . \mathrm{Kw}$

\section{Introduction}

The high purity Fe-Si powder provides an optimal ground for a further development of soft magnetic materials. The sintering of the Fe-Si alloy is carried out entirely in $\alpha$ phase, which leads to a formation of sizable ferrite grains, promoting low coercive force and high permeability [1]. The AFM and MFM techniques have been widely exploited in order to study the topography of sample surface in combination with the visualization of magnetic structure (magnetic domains and walls). In addition, the microstructural-crystallographic technique EBSD can be used to elucidate the texture or preferred orientation of any crystalline or polycrystalline material [2]. This combination of techniques provides a high-image resolution for both scientific explorations and technical applications. Magnetic properties of ferromagnetic materials depend basically on how magnetic domains, which refer to a small region with a parallel orientation of magnetic moments, are spatially arranged. All the magnetic moments within each domain are preferably oriented along the easy axis of magnetization (EAM), related to some specific crystallographic axis. The imaging of magnetic domains then depends on how the EAM of grains is oriented with respect to the perpendicular direction of the MFM tip. The distribution of grains as well as spatial alignment of their crystallographic axes develops during the solidification from the molten state.

In the present work, the topography and magnetic domains of Fe-Si spherical particles will be detected us-

*corresponding author; e-mail: mstreckova@imr.saske.sk ing AFM and MFM techniques. The microstructuralcrystallographic technique EBSD is further used to examine the crystallographic orientation within each individual grain of Fe-Si powder particles. In spite of many reports concerned with MFM images of Fe-Si alloys, it is still highly desirable to confront high-resolution imaging of domain structures with the crystallographic orientation of grains and their boundaries.

\section{Experimental}

The studied spherical Fe-Si particles were obtained from Högänes Corporation in the granulometric fraction from $45 \mu \mathrm{m}$ to $150 \mu \mathrm{m}$ [3]. The cross-sections of powder particles were prepared by grinding and polishing using diamond media. The powder particles were fixed in the epoxy resin. Final polishing was accomplished in the OPS suspension (base suspension with colloid silica grain size under $0.02 \mu \mathrm{m}$ ) for etching of grain boundaries.

AFM and MFM measurements were carried out at room temperature on Dimension Icon ${ }^{\circledR}$ Atomic Force Microscope made by Veeco Instruments (now Bruker, USA) using MESP-LC cantilevers (Bruker AFM Probes, USA). The measurements were executed by two-pass method. In the first pass the topography is determined in the tapping mode. In the second pass the cantilever is lifted to a selected height for each scan line, and scanned using the stored topography (without the feedback). During the second pass the cantilever is affected by long-range magnetic force. Both the height image and the magnetic image were simultaneously obtained within this method. EBSD analysis was performed using Lyra 3 FEG/SEM (Tescan, CZ) equipped by EBSD analyser Nordlys (Oxford Instruments, UK). Kikuchi's patterns of individual points, having dimension of approximately 1-2 $\mu \mathrm{m}$ were obtained. Raw data were then 
indexed and visualised by AZtekHKL software (Oxford Instruments, UK).

\section{Results and discussion}

Figure 1 compares typical AFM topography of one FeSi particle of about $50 \mu \mathrm{m}$ in diameter (Fig. 1a) with the corresponding MFM image of the same particle (Fig. 1b). The grain boundaries are clearly visible from the AFM image as thin lines. It is quite obvious that the investigated particle is split into several grains and each grain has its own domain structure consisting of several magnetic domains. The individual grains evidently have different crystallographic orientations, because different domain structures can be clearly recognized from the MFM image (for instance, the grain on the top of particle has a very different domain structure with respect to others). Two largest grains extending over the left and right part of the Fe-Si particle, have a quite typical maze-like domain structure [2], whereas the largest magnetic domains are of approximate width $1.55 \mu \mathrm{m}$. The maze pattern of magnetic domains is consistent with a rather high strained surface of the Fe-Si particle after the mechanical polishing [2]. The MFM imaging of the aforementioned two largest grains is consistent with the closure domain structure, in which the magnetic moment of individual domains lies on the surface of the Fe-Si particle and the relevant magnetic domains are predominantly separated by $180^{\circ}$ domain walls and less frequently by $90^{\circ}$ domain walls. A more complex domain structure can be seen in the grain on the top of particle.
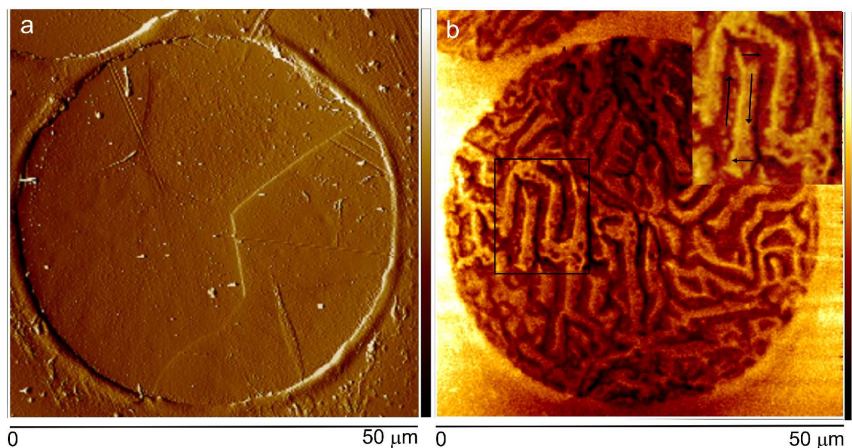

Fig. 1. AFM and MFM images of the same Fe-Si particle. The inset of Fig. 1b shows an inferred orientation of magnetic moment within the closure domain structure.

Figure 2 shows a comparison of the magnetic domain structure (Fig. 2a) with the crystal orientation map (Fig. 2b) of the individual Fe-Si particle. The relevant colour in the EBSD map represents an average misorientation of FCC crystallographic axes, which is expressed by Euler rotational angles within Bunge's notation [4] in each analysed point. The black dots represent points where data was not properly measured or evaluated due to scratches, etc. The crystal orientation map visualised by EBSD analysis, determines the grain structure of Fe$\mathrm{Si}$ particle. It is quite apparent from Fig. $2 \mathrm{a}$ and Fig. $2 \mathrm{~b}$ that the magnetic domain structure basically changes across grain boundaries.

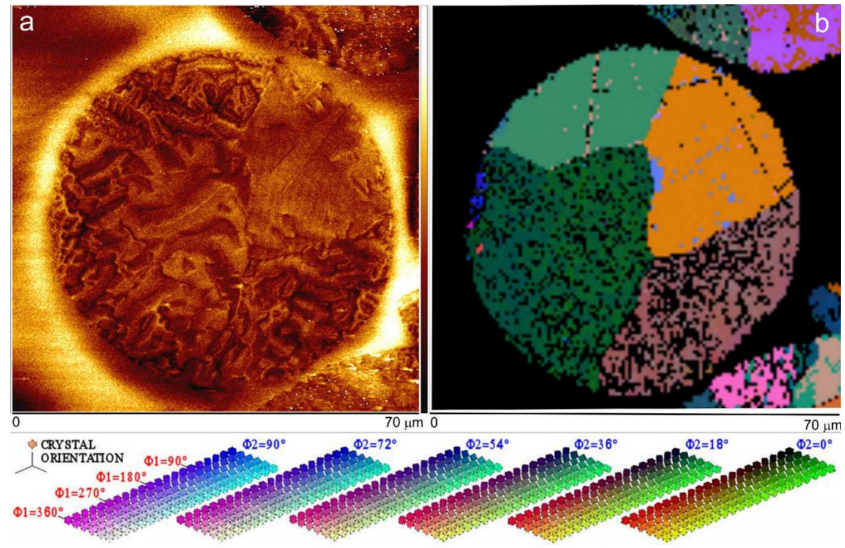

Fig. 2. MFM and EBSD images of one Fe-Si particle. The attached legend determines a colour map for the crystallographic orientation [4].

As a matter of fact, the most substantial changes in the domain structure can be observed at boundary between two grains with the most distinct crystallographic orientations. A more detailed analysis of the crystal orientation map shows that the outer parts of grains possess a slight difference in a crystallographic orientation (change is just in colour shade), which is also reflected in a relevant change of the magnetic domain structure that is basically influenced by the tendency to form the closure domains.

\section{Conclusions}

The AFM studies of the topography, supplemented with the MFM imaging, have demonstrated that the investigated powder of $\mathrm{Fe}-\mathrm{Si}$ particles is formed by grains of diameter about $30 \mu \mathrm{m}$, whereas magnetic domain structure of the grains basically changes across grain boundaries. A supplemental EBSD analysis helped to elucidate how the crystallographic orientation of grains influences the magnetic domain structure.

\section{Acknowledgments}

This work was supported by projects VEGA 2/0155/12, VEGA 2/0184/13 and European Union European Regional Development Fund (ERDF) under the contract no. ITMS 26220120005, the Centre of Excellence CFNT MVEP of the SAS and by the project CZ.1.05/1.1.00/02.0068 CEITEC - Central European Institute of Technology.

\section{References}

[1] J.A. Bas, J.A. Calero, M.J. Dougan, J. Magn. Magn. Mater. 254-255, 391 (2003).

[2] R.M. Bozorth, Ferromagnetism, IEEE Press, 2003.

[3] Högänes datasheet, hoganas.com.

[4] H.J. Bunge, Texture Analysis in Materials Science, Butterworth-Heinemann, 1983. 